\title{
Enhanced carotid-cardiac baroreflex response and elimination of orthostatic hypotension 24 hours after acute exercise in paraplegics
}

\author{
K A Engelke MS, J Darrell Shea MD, D F Doerr BSEE, V A Convertino PhD \\ Biomedical Operations and Research Office, Kennedy Space Center, FL 32899, USA; \\ Lucerne Spinal Center, Humana Hospital Lucerne Orlando, FL 32801, USA; Center for \\ Exercise Science, University of Florida, Gainesville, FL 32611, USA.
}

To test the hypothesis that an acute bout of maximal exercise can ameliorate orthostatic hypotension consequent to prolonged wheelchair confinement, we evaluated heart rate (HR), systolic (SBP) and diastolic (DBP) blood pressure responses during 15 minutes of $70^{\circ}$ head-up tilt (HUT) in 10 paraplegic subjects 24 hours after arm crank exercise designed to elicit maximal effort, and during a control (no exercise) condition. Additionally, the carotid baroreceptor stimuluscardiac response relationship was determined by measurement of $\mathrm{R}-\mathrm{R}$ interval during external application of graded pressures to the carotid sinuses. One week separated the treatment conditions. The maximum slope of the carotid-cardiac baroreflex response was increased $(p=0.049)$ by exercise $(6.2 \pm 1.7$ $\mathrm{msec} / \mathrm{mmHg}$ ) compared to control $(3.3 \pm 0.6)$. During control HUT, HR increased from $61 \pm 1$ to $90 \pm 7 \mathrm{bpm}(p=0.001)$ while SBP decreased from $118 \pm 5$ to $106 \pm 9 \mathrm{mmHg}(p=0.025)$. During HUT 24 hours after exercise, HR increased from $60 \pm 2$ to $90 \pm 4 \mathrm{bpm}(\mathrm{p}=0.001)$, but the reduction in SBP was essentially eliminated $(116 \pm 5$ to $113 \pm 5 \mathrm{mmHg})$. The reduction in SBP during control HUT $(-12.0 \pm 4.6 \mathrm{mmHg})$ was four-fold larger $(p=0.017)$ than during HUT following exercise $(-3.1 \pm 3.9 \mathrm{mmHg})$. DBP during HUT was not altered in either condition. A single bout of intense, dynamic arm crank exercise eliminated orthostatic hypotension in paraplegics. Equal HR response with smaller reduction in SBP during HUT after exercise was consistent with a measured increased sensitivity of the carotid-cardiac baroreflex. These data provide support for the use of maximal arm crank ergometry as a potential treatment for acute management of orthostatic hypotension in patients confined to wheelchairs.

Keywords: baroreflex; blood pressure; exercise; head-up tilt; orthostatic hypotension; paraplegia.

\section{Introduction}

Orthostatic hypotension can limit rehabilitation of patients with spinal injuries confined to bed and/or wheelchairs. ${ }^{1}$ Although the mechanisms underlying this condition remain unclear, the incidence of hypotension and syncope upon standing following bedrest confinement was associated with impaired autonomic function as indicated by attenuation of orthostatic tachycardia and the carotid-cardiac baroreflex response. ${ }^{2} \mathrm{~A}$ similar association between carotid-cardiac vagal baroreflex dysfunction and hypotension has been reported in wheelchair con- fined quadriplegic subjects. ${ }^{3}$ Thus, impairment of cardioacceleration and development of hypotension during orthostatic challenge in spinal injured patients may reflect alterations in autonomic function that occur as an adaptive response to chronic removal from the upright posture.

Acute graded exercise to exhaustion increased carotid baroreceptor-cardiac reflex response by $60 \mathrm{~min}$ post exercise ${ }^{4}$ and lasted as long as 24 hours after exercise. ${ }^{5}$ A single bout of intense exercise at the end of 10 days of bedrest restored heart rate, blood pressures and orthostatic stability on a treadmill 
to prebedrest levels within 2 hours of ambulation ${ }^{6}$ and reversed fainting episodes after 6 hours of water immersion. ${ }^{7}$ These observations suggest that maximal exercise may provide an acute therapeutic procedure against the development of orthostatic hypotension. We therefore hypothesized that a bout of graded exercise designed to elicit maximal effort would increase the sensitivity of autonomic baroreflexes in paraplegics and enhance their blood pressure stability during an orthostatic challenge. Consequently, we measured the carotid-cardiac baroreflex stimulus-response relationship as a marker of autonomic function and responses of heart rate (HR), systolic (SBP) and diastolic (DBP) blood pressures to $70^{\circ}$ head-up tilt (HUT) in 10 paraplegic subjects 24 hours after a bout of dynamic arm exercise. Our results indicated that a single bout of intense exercise eliminated orthostatic hypotension without affecting HR response during HUT in paraplegics and was associated with an increased sensitivity of the carotid-cardiac baroreflex response.

\section{Methods}

\section{Subjects}

Ten sedentary paraplegics (range of injury level: T1-T12) with a mean ( \pm SE) age of $36 \pm 4 \mathrm{yr}$, height of $185 \pm 2 \mathrm{~cm}$, and weight of $90 \pm 7 \mathrm{~kg}$, gave their written consent to serve as subjects for this investigation after they had been informed of all procedures and risks. Subjects had been confined to their wheelchairs for an average of $118 \pm 21$ months and none had attained the upright posture for at least 6 months prior to the beginning of the study. Selection of subjects was dependent on clinical results from a screening evaluation that included a detailed medical history and a physical examination. All subjects were asked to abstain from exercise, tobacco, alcohol, caffeine, and medications not essential for the maintenance of clinical stability for a minimum of 24 hours prior to each experimental session.

\section{Experimental design}

Subjects completed two experimental protocols. Each protocol involved measurement of the carotid-sinus baroreflex sensitivity and responses of HR, SBP and DBP to $70^{\circ}$ HUT on 2 days: (1) 24 hours after acute, intense exercise; and (2) during a control (no exercise) condition. The test days were separated by one week and the two experimental conditions were given in random order.

\section{Experimental protocol}

Each experimental session began with the measurement of body weight with the subject seated in the wheelchair. The subject was then assisted out of the wheelchair and assumed the supine posture on a tilt table for 30 minutes to establish a stabilized baseline HR and BP. After the baseline period, tests were conducted for measurement of the carotid baroreceptor stimuluscardiac response relationship.

\section{Baroreflex measurement}

A neck chamber device previously described in detail ${ }^{8}$ was utilized to evaluate the carotid stimulus-cardiac response relationship of the baroreflex. Briefly, stimuli were delivered to the carotid baroreceptor by a computer-controlled motor-driven bellows which provided pressure steps to a silastic neck chamber covering the area of the carotid arteries. An initial pressure of $40 \mathrm{mmHg}$ was delivered to the chamber and maintained for $4 \mathrm{R}$ waves. With the next $\mathrm{R}$ wave, the pressure sequentially stepped to approximately $25,-5,10,-20,-35,-50$, and $-65 \mathrm{mmHg}$ followed by a return to ambient pressure. Pressure steps were triggered by $\mathrm{R}$ waves so that neck chamber pressures were superimposed upon naturally occurring cardiac pulses. To avoid respiration-related variations of cardiac vagal outflow, neck pressure changes were applied only while subjects held their breath at mid expiration.

A test session consisted of seven successful applications of the aforementioned neck pressure sequences. Each sequence lasted $\sim 15$ seconds and each test session lasted 15 minutes. Individual trials were discarded immediately if the subject breathed during the stimulus sequence or if the neck 
chamber failed to seal adequately. SBP and DBP were measured with a sphygmomanometer prior to the application of the stimulus. Carotid distending pressure (CDP) was calculated as systolic pressure minus neck chamber pressure applied during each heart beat; this calculation assumes complete transfer of pressure in the neck chamber to the carotid arteries. The resulting stimulus-response relationship of the baroreflex was derived by plotting R-R intervals at each pressure step against their respective CDP.

Previous studies using similar techniques have shown that a meaningful fit to a four-parameter logistic equation is not possible for many subjects. ${ }^{9}$ Therefore, from the average of each seven trials, baroreflex relationships were reduced to the following parameters for statistical comparisons: (1) maximum slope to provide an index of reflex sensitivity; (2) position of the operational point ([baseline R-R - minimum $\mathrm{R}-\mathrm{R}] / \mathrm{R}-\mathrm{R}$ range $\times 100 \%$ ) to provide information about the position from which the baseline heart rate functions on the stimulus-response relationship; and (3) carotid pressures at minimum and maximum $\mathrm{R}-\mathrm{R}$ intervals to provide information about threshold and saturation characteristics of the reflex relationship. The maximum slope of the carotid stimulus-cardiac response relationship provides the best indicator of baroreflex function. ${ }^{10}$ To determine the segment of steepest slope, least squares linear regression analysis was applied to every set of 3 consecutive points on the response relationship.

\section{$70^{\circ}$ head-up tilt}

A motorized tilt table elevated the subject to the $70^{\circ}$ head-up position within 10 seconds. Subjects remained in this position for 15 minutes or until the onset of presyncopal symptoms. During this time, beat-tobeat HR was continually recorded with an ECG telemetry system. BP was measured on the right arm via brachial artery ascultation at 2-minute intervals. At the conclusion of the test, the subject was quickly returned to the supine posture. An identical procedure was repeated one week later to measure responses under the remaining treatment.

\section{Exercise bout}

All subjects performed a multistage graded exercise bout designed to elicit a maximal effort 24 hours prior to one of the HUT sessions utilizing a Monark arm crank ergometer mounted on a table capable of being adjusted for the subject's seated height. After a brief (3-5 minutes) warm-up against no load, the work rate was set at 10 Watts (W) and was increased by $5 \mathrm{~W}$ every 2 minutes. An electric metronome assisted the subject in maintaining a rotational cadence of $60-70 \mathrm{rpm}$. This protocol was chosen because similar bouts of exhaustive exercise have been shown to increase baroreflex sensitivity. ${ }^{4,5} \mathrm{HR}$ was recorded during the last 15 seconds of each minute while BP was measured by brachial artery ascultation before and immediately after cessation of exercise. The exercise bout was terminated when the subject reached volitional fatigue and was unable to maintain the required cadence for a period exceeding 15 seconds.

\section{Statistical analysis}

Standard descriptive statistics were performed on each of the response variables of interest with results presented as means \pm SE. Paired-difference $t$ statistics were computed for each variable to compare responses obtained under the two treatment conditions.

\section{Results}

\section{Exercise bout}

For the 10 subjects, the final work rate at volitional fatigue averaged $38 \pm 1 \mathrm{~W}$ and was attained after a mean time of $14 \pm 1$ minutes. HR, SBP and DBP at termination averaged $175 \pm 2 \mathrm{bpm}, 165 \pm 2 \mathrm{mmHg}$, and $71 \pm 1 \mathrm{mmHg}$, respectively.

\section{Baroreflex response}

A summary of the carotid-cardiac baroreflex characteristics for the two experimental 
conditions together with the resultant $t$ and $p$ values is presented in Table I. Supine HR, SBP, DBP, and baseline R-R interval were similar between exercise and control conditions as were the CDP at the maximum R-R interval and the position of the operational point. Exercise did reduce $(p=0.023)$ CDP at minimum $\mathrm{R}-\mathrm{R}$ interval. The maximum slope of the baroreflex was increased ( $p=0.049) 24$ hours following intense exercise $(6.2 \pm 1.7 \mathrm{msec} / \mathrm{mmHg})$ compared to the control condition $(3.3 \pm 0.6 \mathrm{msec} /$ $\mathrm{mmHg}$ ). Mean baroreflex stimulus-response relationships for all subjects during control and after exercise are illustrated in Figure 1 for the purpose of a descriptive comparison.

\section{Responses to $70^{\circ} \mathrm{HUT}$}

Supine SBP, DBP, and HR prior to $70^{\circ}$ HUT were not different between treatments (Table I). During HUT, HR was elevated $(p=0.001)$ by $29 \mathrm{bpm}$ and $30 \mathrm{bpm}$ under control and exercise conditions, respectively (Fig 2a). During control HUT, SBP decreased $(p=0.025)$ from $118 \pm 5$ to $106 \pm 9 \mathrm{mmHg}$ (Fig 2b), while reduced SBP was essentially eliminated during HUT 24 hours after maximal exercise (116 \pm 5 to $113 \pm 5 \mathrm{mmHg}$ ). The reduction in SBP during control HUT $(-12.0 \pm 4.6 \mathrm{mmHg})$ was larger $(p=0.017)$ than that during HUT following exercise $(-3.1 \pm 3.9 \mathrm{mmHg})$. DBP during HUT was not altered in either condition.

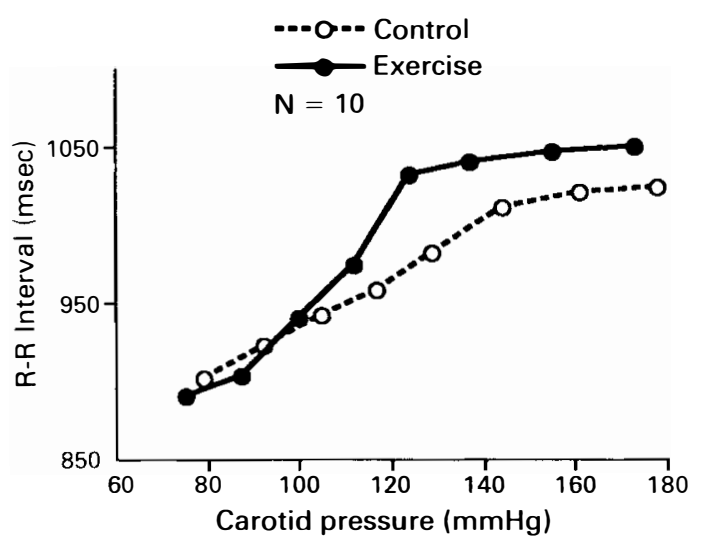

Figure 1 Illustration of carotid baroreceptor stimulus-cardiac response relationship for control (open circles and broken lines) and exercise (solid circles and solid lines) conditions.

\section{Discussion}

To test the hypothesis that acute exercise designed to elicit maximal effort can enhance autonomic function and ameliorate orthostatic hypotension consequent to prolonged wheelchair confinement, we measured the sensitivity of the carotid baroreflex and heart rate and blood pressure responses to 15 minutes of $70^{\circ} \mathrm{HUT}$ in paraplegic subjects 24 hours after intense arm crank exercise. The primary finding of this study was that exercise of this type induced a substantial increase in the maximum slope of the carotid baroreceptor

Table I Heart rate, blood pressure, and parameters of carotid-cardiac baroreflex response relation during control and $24 \mathrm{hr}$ after acute intense exercise

\begin{tabular}{|c|c|c|c|c|}
\hline & Control & Post exercise & $t$ value & $p$ value \\
\hline Supine systolic BP (mmHg) & \pm 5 & $116 \pm 4$ & 0.2459 & 0.811 \\
\hline Supine diastolic BP (mmHg) & $77 \pm 4$ & \pm 4 & 0.5929 & 0.568 \\
\hline $\mathrm{CDP}$ at $\max \mathrm{R}-\mathrm{R}(\mathrm{mmHg})$ & \pm 12 & \pm 9 & 0.2442 & 0.813 \\
\hline $\mathrm{CDP}$ at $\min \mathrm{R}-\mathrm{R}(\mathrm{mmHg})$ & \pm 11 & 85 & 2.7358 & 0.023 \\
\hline Supine HR (bpm) & $61 \pm 1$ & \pm 2 & 0.8316 & 0.427 \\
\hline Baseline R-R interval (ms) & $973 \pm 37$ & $978 \pm 36$ & 0.0695 & 0.946 \\
\hline Maximum slope (ms/mmHg) & $3.3 \pm 0.6$ & $6.2 \pm 1.7$ & 3.7060 & 0.049 \\
\hline Position of operational point ( $\%)$ & $65 \pm 16$ & $65 \pm 18$ & 0.0808 & 0.937 \\
\hline
\end{tabular}

Values are means $\pm \mathrm{SE} ; \mathrm{CDP}=$ estimated carotid distending pressure. 

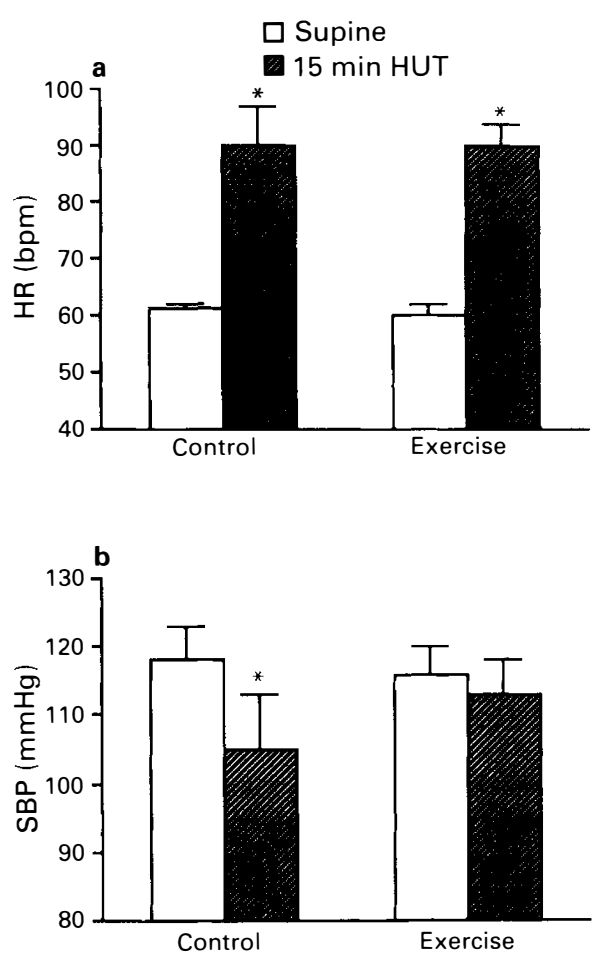

Figure 2 Comparison of heart rate (HR) (panel A) and systolic blood pressure (SBP) (panel B) to the supine posture (open bars) and after 15 minutes of $70^{\circ}$ head-up tilt (HUT) (closed bars) in the control and exercise conditions. Bars represent means and $\mathrm{T}$-lines represent 1 standard error. Asterisks indicate $p<0.05$ vs corresponding supine value.

stimulus-cardiac response relationship and eliminated hypotension during $70^{\circ} \mathrm{HUT}$.

The shape of the baroreflex stimulus-response relationship was shifted upward and to the left 24 hours after exercise so that the increased maximum slope was in the region of hypotension where the capacity to buffer against moment-to-moment changes in blood pressure during orthostatic challenge could be optimized (Fig 1). Exercise also reduced the threshold for cardiac response indicating that the reflex remained active at carotid pressures associated with greater hypotension. We observed essentially no differences between control and exercise conditions in supine HR, SBP, DBP, CDP at the maximal R-R interval (saturation), or in the position of the operational point.
Interestingly, the location of the operational point was higher on the stimulus-response relationship in subjects of the present study $(65 \%)$ compared to a position of $30-40 \%$ reported in other investigations. ${ }^{3,5}$ Because the operational point identifies the location from which the baseline cardiac response functions, the data of the present study suggest that our paraplegic subjects have greater reserve for cardioacceleration during the onset of orthostatic hypotension. Therefore, elimination of orthostatic hypotension following exercise in paraplegic patients was associated with increased maximum slope in the hypotensive range, decreased threshold, and relatively high positioning of the operational point.

Evaluation of the unit change in heart rate per unit change in systolic blood pressure $(\Delta \mathrm{HR} / \Delta \mathrm{SBP})$ during the tilt table test can be useful as a functional assessment of the integrated cardiac baroreflex responsiveness. ${ }^{11}$ In the control condition, a reduction of $12 \mathrm{mmHg}$ in SBP stimulated an increase in heart rate of $29 \mathrm{bpm}$ so that $\Delta \mathrm{HR} / \Delta \mathrm{SBP}$ was 2.4 . Twenty-four hours after maximal arm crank exercise, a reduction of only $3 \mathrm{mmHg}$ in SBP elicited a similar elevation in heart rate $(30 \mathrm{bpm})$ so that $\Delta \mathrm{HR} / \Delta \mathrm{SBP}$ was 10.0 . Thus, exercise induced a four-fold increase in baroreceptor-cardiac reflex response. Since cardiopulmonary barorceptors do not singly contribute to the reflex control of heart rate, ${ }^{12}$ it is reasonable to attribute the increased cardiac reflex response during orthostatic challenge 24 hours after exercise to sensitized baroreflex control. The two-fold increase in carotid-cardiac baroreflex sensitivity concomitant with the four-fold increase in $\Delta \mathrm{HR} / \triangle \mathrm{SBP}$ suggests that acute intense exercise also increased the sensitivity of aortic baroreceptor reflex control of heart rate. This is reasonable since aortic baroreceptor-cardiac reflex has a more prominent role than the carotid baroreceptor-cardiac reflex in the control of heart rate. ${ }^{13,14}$

Our observation that acute intense arm crank eliminated orthostatic hypotension with similar cardioacceleration to that in the control condition suggests that factors other than baroreflex control of heart rate were 
altered by exercise. It is possible that the acute exercise enhanced cardiac filling, stroke volume and cardiac output since a $10 \%$ expansion in plasma volume was induced 24 hours after the performance of this type of exercise in ambulatory subjects. ${ }^{15}$ It is further possible that acute intense exercise might sensitize cardiopulmonary baroreflex control of vascular resistance, although no experiments have addressed this issue.

Our observation of a $95 \%$ increase in sensitivity of the vagally-mediated carotidcardiac baroreflex following a bout of acute high intensity exercise is not without precedent. Somers et $a^{4}$ reported a $50 \%$ increase in the gain of the baroreceptor stimulus-response relationship 60 minutes after the completion of graded cycle exercise to exhaustion in hypertensive subjects. Convertino and Adams ${ }^{5}$ observed a $92 \%$ increase in the baroreflex sensitivity 3 hours after the cessation of maximal leg exercise in normotensive subjects which was maintained for 24 hours. Similarly, a single bout of intense supine exercise following 7 days of bedrest increased the gain of the baroreflex by $32 \% .{ }^{16}$

The mechanisms by which baroreflex function may be sensitized by acute intense exercise is unclear. By using combinations of exercise and lower body positive pressure to increase arterial pressure, the gain of the carotid-cardiac baroreflex response was systematically elevated to $120 \%$ above the normal resting baseline level. ${ }^{17}$ These data were used to advance the hypothesis that loading of carotid baroreceptors by periodic elevation of systolic blood pressure may provide a primary stimulus to increase baroreflex sensitivity. ${ }^{18}$ This hypothesis is supported by the observation that acute elevations in pulse amplitude and frequency in isolated carotid sinuses of dogs increased baroreceptor sensitivity. ${ }^{19}$ If regular transient elevations in arterial pressure are required to maintain baroreflex function, then techniques capable of producing such pressure changes could promote acute sensitization of baroreflex responses. Intense dynamic graded exercise appears to represent one such technique.

A comparison of baroreflex sensitivity between various subject populations is presented in Table II. Quadriplegics and healthy subjects confined to bedrest exhibit baroreflex responses attenuated by $\sim 30 \%$ compared to ambulatory subjects. Paraplegics in the present study exhibited similar baseline resting baroreflex sensitivity as those observed in ambulatory subjects. Further, exhaustive arm exercise performed in the present study by paraplegics increased the gain of the baroreflex stimulus-response relationship to the same magnitude as that induced by maximal supine leg cycle exercise performed by ambulatory subjects in a previous study. ${ }^{5}$ In the present study, arm exercise increased heart rate to $175 \mathrm{bpm}$ and systolic blood pressure to $165 \mathrm{mmHg}$ compared to $174 \mathrm{bpm}$ and $205 \mathrm{mmHg}$ produced by graded supine leg exercise in a previous study. ${ }^{5}$ The observation that arm exercise induced increased baroreflex sensitivity equal in magnitude to that of leg exercise may reflect the capability of both exercises

Table II Comparison of baroreflex sensitivity in ambulatory and confined subjects

\begin{tabular}{llcc}
\hline & & \multicolumn{2}{c}{$\begin{array}{c}\text { Maximum slope } \\
(\mathrm{ms} / \mathrm{mm} \mathrm{Hg})\end{array}$} \\
\cline { 3 - 4 } Study & Subjects & Control & Post exercise \\
\hline Present Study & Paraplegics & $3.3 \pm 0.7$ & $6.2 \pm 1.7$ \\
Convertino et al $^{3}$ & Quadriplegics & $2.6 \pm 0.4$ & $\mathrm{~N} / \mathrm{A}$ \\
${\text { Convertino } \text { et }^{2}}^{2}$ & Bedrested subjects & $2.5 \pm 0.3$ & $\mathrm{~N} / \mathrm{A}$ \\
Convertino \& Adams $^{5}$ & Ambulatory subjects & $3.7 \pm 0.4$ & $6.6 \pm 0.6$ \\
\hline
\end{tabular}

Values are $\pm \mathrm{SE} ; \mathrm{N} / \mathrm{A}=$ not applicable. 
to provide comparable autonomic stimuli as indicated by similar rapid and sustained elevations in heart rate and blood pressure. Thus, graded arm crank exercise may provide an equally effective technique compared to leg exercise for acute improvement of autonomic function associated with blood pressure regulation during orthostatic challenge.

In summary, a single bout of intense graded arm crank exercise increased the sensitivity of the carotid baroreceptor-cardiac reflex response relationship 24 hours after exercise in paraplegic patients. This increased autonomic function was associated with the elimination of orthostatic hypotension during $70^{\circ} \mathrm{HUT}$. These results suggest that this type of exercise may be helpful as a therapeutic intervention designed to counteract orthostatic hypotension in patients chronically confined to bed or wheelchairs.

\section{Acknowledgements}

The authors thank Theresa Nachtscheim for special efforts in recruiting the subjects; Joanne Weisner for her administrative assistance; Susan Parsch, Jill Polet, and Mary Lasley for providing medical monitoring for the subjects during experiments; Marc Duvoisin, Art Maples, and Sandy Reed for engineering support; Dick Triandifils and Barry Slack for their technical support; and the subjects for their cheerful cooperation. This research was supported in part by a NASA grant administered under contract NAS10-11624.

\section{References}

1 Gonzalez F, Chang JY, Banovac K, Messina D, Martinez-Arizala A, Kelley RE (1991) Autoregulation of cerebral blood flow in patients with orthostatic hypotension after spinal cord injury. Paraplegia 29:1-7.

2 Convertino VA, Doerr DF, Eckberg DL, Fritsch JM, Vernikos-Danellis J (1990) Head-down bed rest impairs vagal baroreflex responses and provokes orthostatic hypotension. J Appl Physiol 68: 1458-1464.

3 Convertino VA, Adams WC, Shea JD, Thompson CA, Hoffler GW (1991) Impairment of carotid-cardiac vagal baroreflex in wheelchair-dependent quadriplegics. Am J Physiol (Regulatory Integrative Comp Physiol) 260: R576-R580.

4 Somers VK, Conway J, LeWinter M, Sleight P (1985) The role of baroreflex sensitivity in post-exercise hypotension. J Hypertens 3: S129-S130.

5 Convertino VA, Adams WC (1991) Enhanced vagal response during $24 \mathrm{~h}$ after acute exercise. Am J Physiol (Regulatory Integrative Comp Physiol) 260: R570-R575.

6 Convertino VA (1987) Potential benefits of maximal exercise just prior to return from weightlessness. Aviat Space Environ Med 58: 568-572.

7 Stegemann J, Meier U, Skipka W, Hartlieb W, Hemme B, Tibes U (1975) Effects of multi-hour immersion with intermittent exercise on urinary excretion and tilt table tolerance in athletes and nonathletes. Aviat Space Environ Med 46: 26-29.

8 Sprenkle JM, Eckberg DL, Goble RL, Schelhorn JJ, Halliday HC (1986) Device for rapid quantification of human carotid baroreceptor-cardiac reflex responses. J Appl Physiol 60: 727-732.

9 Kasting GA, Eckberg DL, Fritsch JM, Birkett CL (1987) Continuous resetting of the human carotid baroreceptor-cardiac reflex. Am J Physiol (Regulatory Integrative Comp Physiol) 252: R737-R736.

10 Ludwig DA, Convertino VA (1991) A statistical note on the redundancy of nine baroreflex parameters. Aviat Space Environ Med 62: 172-175.

11 Smith ML, Graitzer HM, Hudson DL, Raven PB (1988) Baroreflex function in endurance- and static exercise-trained men. J Appl Physiol 64: 585-591.

12 Mark AL, Mancia G (1983) Cardiopulmonary baroreflexes in humans. In: Shepherd JT, Abboud FM, editors. Handbook of Physiology, Section 2, The Cardiovascular System, Vol III, Peripheral Circulation and Organ Blood Flow, Part 2. American Physiological Society, Bethesda, MD: 795-813.

13 Ferguson DW, Abboud FM, Mark AL (1985) Relative contribution of aortic and carotid baroreflexes to heart rate control in man during steady-state and dynamic increases in arterial pressure. J Clin Invest 76: 2265-2274.

14 Mancia G, Ferrari A, Gregorini L, Valentini R, Ludbrook J, Zanchetti A (1977) Circulatory reflexes from carotid and extra carotid baroreceptor areas in man. Circ Res 41: 309-315.

15 Gillen CM, Lee R, Mack GW, Tomaselli CM, Nishiyasu T, Nadel ER (1991) Plasma volume expansion in humans after a single intense exercise protocol. J Appl Physiol 71: 1914-1920.

16 Convertino VA, Doerr DF, Guell A, Marini JF (1992) Effects of acute exercise on attenuated vagal baroreflex function during bedrest. Aviat Space Environ Med 63: 999-1003.

17 Eiken O, Convertino VA, Doerr DF, Dudley GA, Morariu G, Mejkavic IB (1992) Characteristics of the carotid baroreflex in man during normal and flow-restricted exercise. Acta Physiol Scand 144: 325-331. 
18 Convertino VA (1992) Effects of exercise and inactivity on intravascular volume and cardiovascular control mechanisms. Acta Astronautica 27: 123-129.

19 Chapleau MW, Abboud FM (1989) Determinants of sensitization of carotid baroreceptors by pulsatile pressure in dogs. Circ Res 65: 566-577. 